\title{
Team- und Projektarbeit in der digitalisierten Produktentwicklung
}

\author{
Victoria Zorn, Julian Baschin, Nine Reining, David Inkermann, \\ Thomas Vietor und Simone Kauffeld
}

\subsection{Einführung}

Die fortschreitende Globalisierung und Digitalisierung verändern die Arbeitswelt (Franken 2016). Technologische Innovationen bieten bereits seit längerer Zeit Möglichkeiten zur standortverteilten, virtuellen Zusammenarbeit, um beispielsweise Fachkräfte ortsunabhängig anzuwerben und zu beschäftigen (Lipnak und Stamps 1998) oder Zusammenarbeitsformen und Prozesse zu flexibilisieren (Hirsch-Kreinsenet al. 2015).

V. Zorn $(\bowtie) \cdot$ N. Reining $\cdot$ S. Kauffeld

Abteilungs für Arbeits-, Organisations- und Sozialpsychologie, Institut für Psychologie,

Technische Universität Braunschweig, Braunschweig, Deutschland

E-Mail: v.zorn@tu-bs.de

N. Reining

E-Mail: n.reining@tu-bs.de

S. Kauffeld

E-Mail: s.kauffeld@tu-braunschschweig.de

J. Baschin · T. Vietor

Universität Braunschweig, Institut für Konstruktionstechnik, Technische,

Braunschweig, Deutschland

E-Mail: j.baschin@tu.bs.de

T. Vietor

E-Mail: t.vietor@tu-braunschweig.de

D. Inkermann

Technische Universität Clausthal, Institut für Maschinenwesen,

Clausthal-Zellerfeld, Deutschland

E-Mail: inkermann@imw.tu-clausthal.de

(C) Der/die Autor(en) 2021

S. Mütze-Niewöhner et al. (Hrsg.), Projekt- und Teamarbeit in der digitalisierten

Arbeitswelt, https://doi.org/10.1007/978-3-662-62231-5_8 
Zudem wird durch Automatisierung, Cyber-Physische Systeme oder Werkerassistenzsysteme die Ausführung von physischer Arbeit unmittelbar verändert (Blumberg und Kauffeld 2020; Lanting und Lionetto 2015).

Für den Anlagen- und Maschinenbau ergeben sich daraus nicht nur Veränderungen darin, wie gearbeitet wird, sondern auch eine zunehmende Komplexität der zu entwickelnden Produkte, da Hardware wie Maschinen oder Anlagen mit Software kombiniert werden müssen (Eigner et al. 2014). Zugleich bietet sich dadurch die Gelegenheit zur digitalen Erweiterung bestehender Produkte und Dienstleistungen (Born 2018), beispielsweise durch die Verwendung von Sensordaten zur Steuerung von Produktionsprozessen (Lanting und Lionetto 2015). Um dies bewerkstelligen zu können, findet Produktentwicklung häufig in interdisziplinären Projektteams statt, die durch Strukturen und Technologien in ihrer zunehmend komplexen Zusammenarbeit unterstützt werden müssen (Bavendiek et al. 2018; Badke-Schaub und Frankenberger 2004). Digitalisierungsbedingte Veränderungen beschränken sich also nicht nur auf das zu entwickelnde Produkt und die zur Verfügung stehenden bzw. genutzten technologischen Hilfsmittel, sondern auch Arbeitsprozesse und soziale Aspekte von Arbeit sind betroffen (Paulsen et al. 2020; Sträter und Bengler 2019).

Im Alltag stellt sich dann die Produktentwicklung beispielsweise wie folgt dar: Ein Kunde bestellt eine Maschine und nach Auftragsklärung durch die Vertriebsabteilung beginnt die Produktentwicklung. Dazu entwickeln Mitarbeitende aus verschiedenen Fachdisziplinen die einzelnen Teilkomponenten der Maschine wie Hydraulik, Elektronik oder Steuerungssoftware. Die Mitarbeitenden nutzen verschiedene Technologien zur Erfüllung ihrer Aufgaben, z. B. CAx-Systeme (CA: Computer Aided) zur Erstellung von digitalen 3D-Modellen, Simulationen und der Produktionsplanung oder Kommunikationsmedien wie E-Mails oder Telefonanrufe zur Abstimmung mit anderen Abteilungen. Im Laufe des Produktentwicklungsprozesses ergeben sich Änderungen, z. B. Modifizierungen am Design, um die Funktionsfähigkeit zu optimieren oder auf nachträgliche Kundenanforderungen zu reagieren. Auch können sich zwischen Produktentwicklungsprojekten externe Änderungen ergeben, bspw. hinsichtlich gesetzlicher Regelungen oder der Nachfrage bestimmter Produkte oder Produktfunktionalitäten am Markt. Diese Änderungen müssen dann in den darauffolgenden Projekten berücksichtigt werden, sodass Prozesse und Tätigkeiten immer wieder angepasst werden müssen.

Die Produktentwicklung weist damit die wichtigsten Merkmale eines soziotechnischen Systems auf: Um die Entwicklung eines Produktes (primäre Arbeitsaufgabe) leisten zu können, arbeiten Mitarbeitende mit Technik (soziales und technisches Teilsystem) unter komplexen Bedingungen (Offenheit gegenüber der Umwelt) und müssen immer wieder auf Änderungen reagieren (Unvollkommenheit des Systems) (Maguire 2014; Ulich 2013). Für soziotechnische Systeme ist wiederum eine ganzheitliche Betrachtung notwendig, um der Komplexität des Systems gerecht zu werden und passende Lösungen zu finden (Davis et al. 2014; Maguire 2014). Werden nur einzelne Aspekte berücksichtigt, ist das Risiko sehr hoch, erfolgskritische Wechselwirkungen zu übersehen (Davis et al. 2014). Da Produktentwicklung als soziotechnisches System 
zu verstehen ist (Crowder et al. 2003), sind zur Beschreibung, Analyse und Gestaltung Modelle notwendig, die Produktentwicklung möglichst ganzheitlich abbilden (Bavendiek et al. 2018).

Speziell für die Produktentwicklung gibt es jedoch nur sehr wenige solche Ansätze (Bavendiek et al. 2018; Wallace et al. 2001). Bisher lag der Fokus eher auf einzelnen Aspekten wie z. B. Unterstützung durch Informations- und Kommunikationstechnologien (IKT; Talas et al. 2017) oder Wissensverteilung (Robin et al. 2007). Diese Ansätze berücksichtigen in der Regel auch die Mitarbeitenden (z. B. Robin et al. 2007) und erfassen teilweise auch deren Perspektive in Bezug auf prozess- oder technologieorientierte Aspekte (z. B. Crowder et al. 2003). Systematische, umfassende Betrachtungen mit dem Detaillierungsgrad soziotechnischer Systemanalysen (z. B. der MTO-Analyse nach Ulich 2013) sind dagegen selten. Vor dem Hintergrund der digitalisierungsbedingten, zunehmenden Komplexität in der Produktentwicklung besteht daher ein Bedarf an ganzheitlicheren Modellen, die sowohl die relevanten Elemente (z. B. Technologien, Prozesse, Mitarbeitende) als auch deren Interaktion beschreiben. Nur so können Veränderungen in ihrer Komplexität analysiert und erfolgreich gestaltet werden.

Hier setzt das BMBF- und ESF-geförderte Verbundprojekt „KAMiiSo: Digitale Hilfsmittel für Kommunikation und Methodeneinsatz in der standortübergreifenden Produktentwicklung“ an. Ziel des Projektes ist es, kleinen und mittelständischen Unternehmen aus dem Anlagen- und Maschinenbau mit Hilfsmitteln, Methoden und IT-Tools bei der interdisziplinären, standortverteilten Produktentwicklung zu unterstützen. Durch die Digitalisierung bieten sich in diesem Bereich eine Vielzahl neuer Möglichkeiten, von virtuellem Prototyping (Horváth et al. 2010) bis hin zu komplexen, kollaborativen Entwicklungsaktivitäten mittels IKT (Talas et al. 2017). Um Nutzen und Konsequenzen von einzelnen Digitalisierungsvorhaben einschätzen zu können, braucht es ein fundiertes, anwendbares Modell, das durch eine ganzheitliche Betrachtung Einblicke in möglichst viele potenziell betroffene Bereiche erlaubt.

\subsection{Das 3-Sichten-Modell}

Dem Verbundprojekt liegt ein Modell zugrunde, das eine ganzheitliche Betrachtung von Entwicklungsaktivitäten anstrebt. Als Ausgangspunkt wurden etablierte Ansätze zur Strukturierung von Produktentwicklungsprojekten in Teilsysteme (z. B. Browning et al. 2006; Negele et al. 1997) aufgegriffen. Diese Ansätze fokussieren häufig nur den Zielzustand und dies zudem nur auf übergeordneter Ebene, bspw. indem organisationale Strukturen, Mitarbeitende und Ressourcen als ein Teilsystem zusammengefasst werden (Negele et al. 1997). Dies erschwert die praxisbezogene Nutzung solcher Ansätze, weil Status Quo und damit Abweichungen vom Zielzustand nicht berücksichtigt werden. Darüber hinaus müssen konkrete Analyse- und Gestaltungskriterien zur Anwendung dieser Modelle jeweils abgeleitet werden, da die übergeordnete Betrachtungsebene 
kaum spezifische Kriterien bereitstellt. Aus diesem Grund wurde die soziotechnische Systemperspektive aufgegriffen, die Arbeitseinheiten als komplexes System betrachtet, das aus verschiedenen Teilsystemen besteht (z. B. Davis et al. 2014; Maguire 2014). Den Ansätzen aus der Produktentwicklung und dem soziotechnischen Systemansatz ist gemein, dass sie eine ganzheitliche Betrachtung fordern, um Veränderungen und ihre Auswirkungen möglichst umfassend berücksichtigen zu können (z. B. Maguire 2014; Negele et al. 1997). Die soziotechnische Systemperspektive betrachtet aber neben dem Zielzustand auch den Ausgangszustand eines Systems und bietet bereits Analysevorgehen (z. B. MTO-Analyse n. Ulich2013) sowie konkrete Gestaltungskriterien (z. B. Pasmore et al. 2019).

Aus diesen Überlegungen entstand das 3-Sichten-Modell, das möglichst konkret die Analyse, Beschreibung und Gestaltung von Produktentwicklungsprojekten ermöglichen soll. Das 3-Sichten-Modell betrachtet daher Entwicklungsaktivitäten auf Prozess-, Methoden- und Kompetenzebene (s. auch Abb. 8.1; Bavendiek et al. 2017). Die Prozesssicht betrachtet dabei speziell Aspekte des Ablaufs und der Organisation. Ein Prozess ist dabei eine Abfolge von einzelnen Aktivitäten, die miteinander in Verbindung stehen, um ein Ziel zu erreichen. Zusätzlich zu Aktivitäten werden auch Informationsflüsse und Stakeholder mit betrachtet. Die Methodensicht fokussiert neben Methoden (z. B. Fehlermöglichkeits- und Einflussanalysen, kurz FMEA) und Hilfsmitteln (z. B. Checklisten) auch Technologien, die die Tätigkeit der Mitarbeitenden im Entwicklungsprozess unterstützen können. Diese Technologien und Werkzeuge befassen sich nicht nur mit fachlich notwendigen (z. B. CAx-Systeme) sondern auch mit solchen zur Kommunikation und Kollaboration. Die Kompetenzsicht betrachtet fachliche und überfachliche Kompetenzen und Qualifikationen der beteiligten Mitarbeitenden. Sowohl Prozessschritte als auch Methoden und Technologien verlangen einerseits bestimmte

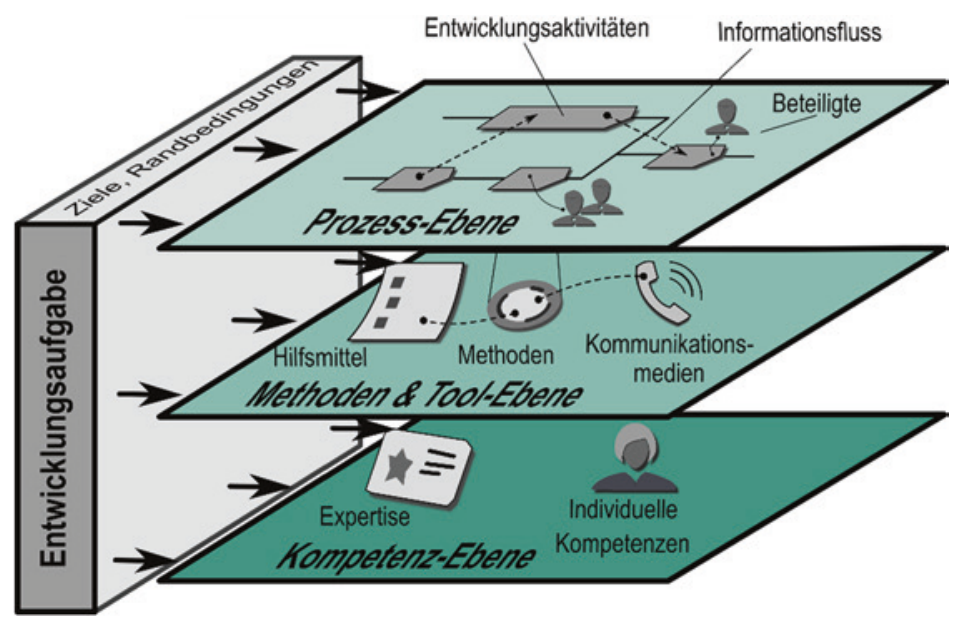

Abb. 8.1 3-Sichten-Modell der Produktentwicklung. (in Anlehnung an Bavendiek et al. 2017) 
(fachlich-methodische) Kompetenzen und Qualifikationen von den Mitarbeitenden, andererseits fordert die Zusammenarbeit mit anderen Teammitgliedern überfachliche Kompetenzen wie bspw. Kommunikationskompetenzen oder Zeitmanagement (s. Kauffeld und Paulsen 2018). Die Interaktionen innerhalb der einzelnen Sichten sowie zwischen den Sichten werden in diesem Modell explizit adressiert (Bavendiek et al. 2017, 2018). Die jeweilige Entwicklungsaufgabe stellt dabei einen wichtigen Rahmen dar und beinhaltet projektspezifischen Vorgaben wie Ziel (z. B. Produkt oder Produkteigenschaften, Verwendungszweck usw.) oder Randbedingungen (z. B. Deadlines, spezielle Anforderungen o. ä.). Da diese Entwicklungsaufgabe einerseits für jedes Produktentwicklungsprojekt spezifisch ist und andererseits zum großen Teil im Kundenbzw. Arbeitsauftrag beschrieben ist, steht die Entwicklungsaufgabe selbst für diesen Beitrag nicht im Fokus.

Für die tatsächliche Anwendung eines Modells in der Praxis ist entscheidend, dass das Modell selbst nicht $\mathrm{zu}$ abstrakt ist. Eine große Herausforderung für den Transfer in die Praxis ist häufig, dass Modelle zu allgemein sind und daher die Realität nicht mehr ausreichend konkret beschreiben können (z. B. Ehrlenspiel und Meerkamm 2013; Inkermann 2019). Dies lässt sich beispielsweise im modellbasierten Systems Engineering beobachten, wenn standardisierte Modelle und Notationen Elemente von Produkten oder Prozessen so generalisiert darstellen, dass eine konkrete Beschreibung derselben in einem Entwicklungsprojekt unter Verwendung der Standardisierung nicht mehr möglich ist (s. Inkermann 2019). Daraus ergibt sich für das 3-Sichten-Modell folgende grundlegende Fragestellung: Inwiefern ist das 3-Sichten-Modell geeignet, um Forschungs- und Entwicklungsprojekte zu gestalten und Mitarbeitende in der Projektarbeit zu unterstützen?

Obwohl dieses Modell bereits sehr umfassend ist, hat sich im Laufe des Verbundprojektes Ergänzungsbedarf gezeigt. Prozesse, Methoden, Technologien und Kompetenzen wurden bisher nur in Bezug auf die Aufgabe der Produktentwicklung betrachtet. Dies vernachlässigt aber einen zentralen Aspekt: Das Team, das die Aufgabe ausführt. Hieraus ergab sich daher im Laufe des Verbundprojektes die ergänzende, theoretisch-konzeptionelle Fragestellung, wie die Teamperspektive im 3-Sichten-Modell berücksichtigt werden kann.

Ziel dieses Beitrags ist es daher, das 3-Sichten-Modell um die Teamperspektive zu erweitern sowie Anwendung und Nutzen dieses Modells mithilfe von zwei Fallbeispielen zu verdeutlichen. Diese Fallbeispiele stammen von zwei Unternehmenspartnern aus dem Anlagen- und Maschinenbau, die im Zuge der Digitalisierung Veränderungen für ihre Produktentwicklung geplant und erprobt haben. Teamarbeit findet bei beiden Unternehmen im Rahmen von Projektteams statt. Mitarbeitende werden dazu je nach notwendigen Kompetenzen und Verfügbarkeit zu Projektteams zusammengestellt und bearbeiten dann als Team ein Produktentwicklungsprojekt zusammen. Während sich die genaue Zusammensetzung pro Team ändern kann, ist die Gesamtzahl an Mitarbeitenden in den beteiligten Abteilungen begrenzt und die Betriebszugehörigkeit der Mitarbeitenden beträgt meist mehrere Jahre. Selbst in einem neu zusammengesetzten 
Projektteam ist die Wahrscheinlichkeit also eher hoch, dass die Teammitglieder vorher bereits zusammengearbeitet haben.

Die Fragestellung zur Berücksichtigung der Teamperspektive wird im Folgenden zuerst adressiert, um nachfolgend die Anwendung des 3-Sichten-Modells sowie die dazugehörigen Ergebnisse für die Lesenden zusammenhängend darzustellen.

\subsection{Teams in der Produktentwicklung}

Für die Entwicklung moderner Maschinen und Anlagen sind Wissen und Kompetenzen einer einzelnen Person längst nicht mehr ausreichend (Albers et al. 2009; Bavendiek et al. 2017). Produktentwicklung findet daher zunehmend in der Zusammenarbeit verschiedener Akteure und Disziplinen statt (engl. „collaborative design“, Bavendiek et al. 2017, S. 350; Huth und Vietor 2020). Dazu werden einerseits Erkenntnisse und Kompetenzen aus verschiedenen Fachdisziplinen kombiniert (z. B. Maschinenbau und Softwareentwicklung), um Funktionalitäten zu erweitern, und andererseits wird standortübergreifend gearbeitet, um die Auslastung der Mitarbeitenden an variierende Auftragslagen anzupassen (Franken 2016; Huth und Vietor 2020).

Dies trägt zwar zur Bewältigung der Komplexität in Bezug auf die primäre Arbeitsaufgabe, d. h. die Entwicklung neuer Produkte, bei, steigert aber zugleich die Gesamtkomplexität im Arbeitssystem. Produktentwicklungsteams werden zwar in der Regel so zusammengesetzt, dass alle notwendigen Kompetenzen zur Erfüllung der fachlichen Anforderungen im Team vorhanden sind, sehen sich aber gleichzeitig mit zunehmenden Kommunikations- und Koordinationsaufgaben konfrontiert (Schleidt und Eigner 2010) und müssen im Falle standortübergreifender Zusammenarbeit auch mit Herausforderungen virtueller Zusammenarbeit umgehen (für eine Zusammenfassung siehe z. B. Boos et al. 2016; Kauffeld et al. 2016; Schulze und Krumm 2017). Dies zeigt sich auch in den gestiegenen Anforderungen an überfachliche, d. h. persönliche und soziale, Kompetenzen von Ingenieurinnen und Ingenieuren in der Produktentwicklung (Albers et al. 2009).

In bisherigen Betrachtungen wurden Teams als Form der Arbeitsorganisation in der Produktentwicklung zwar angenommen (z. B. Robin et al. 2007; Törlind und Larsson 2002), aber nicht als eigene Betrachtungsebene weiterverfolgt. Aus der Teamforschung ist aber bekannt, dass Teams zwischen Individuen und Organisationen eine unterscheidbare Ebene darstellen, die die ersteren beiden miteinander verknüpft (Cummings und Worley 2009).

$\mathrm{Zu}$ Teams liegen bereits umfangreiche Forschungsbefunde vor, die von allgemeinen Fragestellungen wie z. B. Formierungsprozesse in Teams (z. B. Tuckman 1965) bis hin zu spezialisierten Betrachtungen von Teams in bestimmten Kontexten (z. B. High Responsibility Teams; Hagemann et al. 2011) reichen. Für die Erweiterung des 3-Sichten-Modells der Produktentwicklung wurden zwei Aspekte von Teams ausgewählt: Teamkognition und Teamemotion. 


\subsubsection{Teamkognitionen - Geteilte mentale Modelle}

Ein mentales Modell stellt generell eine Form von strukturiertem Wissen dar, das Menschen dazu benutzen, Ereignisse in ihrer Umwelt für sich zu beschreiben, zu erklären und Vorhersagen zu ihnen zu treffen (Rouse und Morris 1986). Geteilte mentale Modelle erweitern dies auf die Teamebene (vgl. Cannon-Bowers et al. 1993). Stimmen die mentalen Modelle der Teammitglieder überein, sind sie in der Lage, die Handlungen anderer Teammitglieder implizit vorherzusagen und ihre eigenen Handlungen daran anzupassen (Mathieu et al. 2000). Demnach benötigen Teammitglieder ein geteiltes Verständnis von ihrer Aufgabe und von ihrem Team, um komplexe Aufgaben effizient zu lösen, insbesondere wenn kaum Möglichkeit zur Abstimmung besteht (Ellwart et al. 2015; Mathieu et al. 2000).

Dabei ist zu beachten, dass geteilte mentale Modelle nicht allumfassend sind, sondern sich auf einen spezifischen Gegenstand beziehen, insbesondere im Teamkontext (CannonBowers et al. 1993). Teams benötigen dabei insgesamt vier Typen geteilter mentaler Modelle, um erfolgreich arbeiten zu können (s. Cannon-Bowers et al. 1993): Modelle zur Aufgabe (z. B. zu Prozessen, Problemen, Lösungsstrategien), zur verfügbaren Ausrüstung (z. B. zu Funktionsweise von Technologien, vorhandene Hilfsmittel), zur Interaktion im Team (d. h. Rollen, Verantwortlichkeiten, Informationsflüsse etc.) sowie zu den einzelnen Teammitgliedern (z. B. zu Kompetenzen, Ansichten usw.). Forschungsbefunde sprechen dafür, dass geteilte mentale Modelle über Teamprozesse einen positiven Einfluss auf die Teamleistung haben (Mathieu et al. 2000). Geteilte mentale Modelle entwickeln sich zudem im Laufe der Zeit, sind also dynamisch und damit potenziell mit Hilfe von Interventionen zu fördern und zu gestalten (z. B. Ellwart et al. 2015).

Die einzelnen Modelltypen lassen sich direkt im 3-Sichten-Modell einordnen: Die Prozessebene korrespondiert mit dem mentalen Modell zur Aufgabe. Die Methodenebene weist Überschneidungen mit dem mentalen Modell zu Ausrüstung auf. Das mentale Modell zu den Teammitgliedern hat Gemeinsamkeiten mit der Kompetenzebene. Und das Modell zur Teaminteraktion bezieht sich auf Wechselwirkungen und Schnittmengen der drei Sichten (z. B. Rollen im Prozess, präferierte Kommunikationskanäle etc.). Mittels des 3-Sichten-Modells können also geteilte mentale Modelle gefördert werden, indem eine Analyse auf Basis des 3-Sichten-Modells die gleiche Wissensgrundlage für alle Teammitglieder schaffen kann. Über kognitive Abgleichung und Anpassung der mentalen Modelle können sich die mentalen Modelle der einzelnen Teammitglieder einander annähern.

\subsubsection{Teamemotion}

Teamemotion (engl. group oder team affect) bezeichnet die affektive Stimmung, positiv oder negativ, innerhalb eines Teams. In Bezug auf Teamleistung leistet Teamemotion einen eigenständigen Beitrag, der nicht mit kognitiven Prozessen wie bspw. mentalen 
Modellen erklärt werden kann (Barsade 2002). Teamstimmung ist übertragbar in dem Sinne, dass Emotionen einzelner Teammitglieder sich als gleichgerichtete Stimmung bei den anderen Teammitgliedern niederschlägt (Cheshin et al. 2011). So können Ärger oder Freude eines Teammitglieds bei den anderen Mitgliedern unbestimmte Gereiztheit oder eine positive Grundstimmung auslösen. In Meetings kann sich dies z. B. in Jammerspiralen oder Lösungszirkeln niederschlagen (Kauffeld 2007). Teamstimmung ist also nicht einfach die durchschnittliche Stimmung der Teammitglieder, sondern entsteht dynamisch im Team über die Zeit und durch Übertragungsprozesse, die sich dann in der Stimmung der einzelnen Teammitglieder zeigt (Barsade 2002).

Inwiefern die Stimmung einzelner im Team übertragen wird, hängt von verschiedenen Faktoren ab. Beispielsweise hängt höhere Aufgabeninterdependenz mit höherer Übertragung von Stimmung zusammen, möglicherweise weil dies die Einheit im Team bei hoher aufgabenbezogener Abhängigkeit fördert (Bartel und Saavedra 2000). Erste Erkenntnisse deuten ebenfalls darauf hin, dass sich überwiegend textbasierte Kommunikation negativ auf die Teamstimmung auswirken kann (Cheshin et al. 2011). Forschungsbefunde zeigen, dass eine positive Teamstimmung indirekt die Teamleistung erhöhen kann, Effekte von negativer Teamstimmung sind dagegen kontextspezifisch, d. h. externe Ursachen für negative Stimmung können den Zusammenhalt im Team fördern, teaminterne Ursachen (z. B. Konflikte) sind dagegen eher schädlich (Knight und Eisenkraft 2015). Ähnliche Effekte finden sich für die Länge der Zusammenarbeit. Zu Beginn der Zusammenarbeit oder bei nur kurz bestehenden Teams kann negative Stimmung Zusammenhalt und Leistung begünstigen, bei länger zusammenarbeitenden Teams treten dagegen eher negative Effekte auf (Knight und Eisenkraft 2015). Des Weiteren kann sich Teamemotion dynamisch über die Zeit verändern, wobei diese Veränderungen ebenfalls mit einer Veränderung in der Teamleistung einhergehen können (Paulsen et al. 2016).

Teamemotion kann sich zudem auch auf Technologieakzeptanz auswirken. Reagieren Mitarbeitende positiv auf neue Technologien, ist deren langfristige Nutzung wahrscheinlicher, während eine negative Einstellung die Implementierung erheblich hemmen kann (Bondarouk und Sikkel 2007). Da Teamemotion sich im Verlauf der Zeit ändern kann, ist es daher für Technologieeinführungen ggf. wichtig, diesen Stimmungsverlauf zu überwachen, um bei negativen Reaktionen auf die Technologie schnellstmöglich eingreifen zu können. Dies ist für den Kontext dieses Beitrags dahin gehend relevant, da die Einführung neuer Technologien jeweils Bestand der Fallbeispiele sind.

Während Teamstimmung nicht direkt einzelnen Teilen des 3-Sichten-Modells zugeordnet werden kann, ist sie doch ein wichtiges Kriterium zur Analyse und Gestaltung, um langfristig höhere Arbeitsleistung sicherzustellen und Differenzen innerhalb des Entwicklungsteams frühzeitig festzustellen.

Insgesamt stellen Teamkognition und Teamemotion daher für den Kontext der Fallbeispiele eine sinnvolle erste Erweiterung des 3-Sichten-Modells dar. Eine positive Ausprägung dieser beiden Merkmale, d. h. gute geteilte mentale Modelle und positive Teamstimmung, können zum Erfolg des Teams beitragen. Beide Faktoren beschreiben zudem emergente Phänomene und bieten damit Ansatzpunkte für Gestaltung im Verlauf 


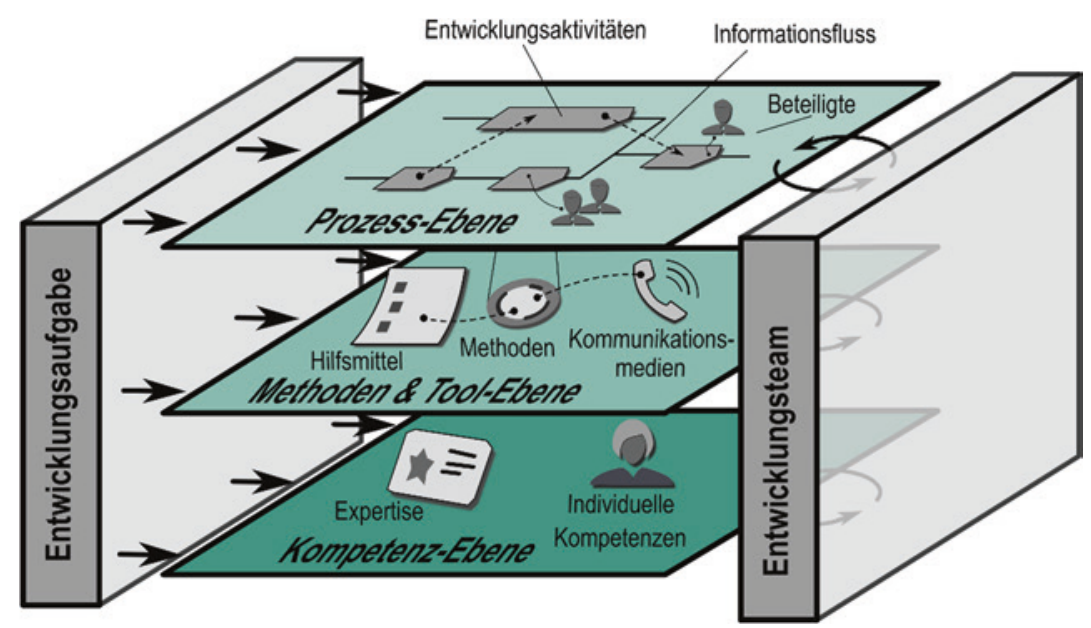

Abb. 8.2 Erweitertes 3-Sichten-Modell inkl. Berücksichtigung des Entwicklungsteams

von Projekten. Die Bildung geteilter mentaler Modelle kann z. B. durch das 3-SichtenModell implizit gefördert werden, weil wichtige Informationen für alle Teammitglieder zugänglich gemacht werden. Teamemotion hingegen kann als Indikator für die Akzeptanz neuer Technologien dienen und so frühzeitig Interventionsbedarf aufzeigen, um den Erfolg des Digitalisierungsvorhaben abzusichern.

Für das 3-Sichten-Modell stellt das Entwicklungsteam zusammen mit der Entwicklungsaufgabe einen wichtigen Rahmen für Produktentwicklungsprojekte dar. Die Entwicklungsaufgabe gibt Inhalt und organisatorische Rahmenbedingungen wie Deadlines o. ä. für ein Projekt vor. Das Entwicklungsteam stellt die umsetzenden Personen dar, die miteinander interagieren, um ein Produkt zu entwickeln. Zusammengenommen beeinflussen Aufgabe und Team, welche Abläufe auf Prozessebene am effizientesten sind, welche der zur Verfügung stehenden Methoden und Tools zum Einsatz kommen und welche Kompetenzen und Expertise notwendig zur Umsetzung sind. Die entsprechende Erweiterung des 3-Sichten-Modells um die Teamperspektive ist in Abb. $8.2 \mathrm{zu}$ sehen.

\subsection{Anwendung des 3-Sichten-Modells in Unternehmen}

Die Anwendung des 3-Sichten-Modells teilte sich in drei Phasen auf: 1) Erfassung des Ist- und Soll-Zustands, 2) Planung des Soll-Zustands und 3) Begleitung von Pilotprojekten zur Annäherung an den Soll-Zustand. Die methodischen Zugänge zu den einzelnen Phasen werden in den folgenden Absätzen kurz skizziert. Die starke Relevanz des Teamkontextes hat sich insbesondere bei der Planung der Pilotprojekte gezeigt, sodass zu Beginn das ursprüngliche 3-Sichten-Modell als Grundlage genutzt wurde und die Erweiterung auf die Teamebene gegen Ende der zweiten Phase stattfand. 
Die Erfassung des Ist- und Soll-Zustandes wurde in Anlehnung an die MTO-Analyse (n. Ulich 2013) durchgeführt. Dabei wurde das 3-Sichten-Modell als Grundlage genutzt und ein Vorgehen in Anlehnung an die ersten Schritte der MTO-Analyse auf Ebene des Unternehmens, der Arbeitsprozesse, der Arbeitssysteme und der Arbeitsgruppen gewählt. Dokumentenanalysen, Interviews mit Mitarbeitenden, Führungskräften und der Geschäftsführung sowie Hot Spot-Analysen wurden als Datenbasis genutzt, um Ist- und Soll-Zustand gemeinsam zu erfassen (für eine detaillierte Beschreibung s. Paulsen et al. 2020). Für den Ist-Zustand stand die Analyse im Vordergrund, während für den SollZustand eine Definition des Idealzustandes fokussiert wurde.

Die Planung des Soll-Zustandes erfolgte dann in einem weiteren Schritt unter Berücksichtigung des 3-Sichten-Modells. Zur Vorbereitung wurden auf Basis der Ergebnisse aus der ersten Phase sowie des 3-Sichten-Modells Gestaltungsempfehlungen für mögliche Pilotprojekte zusammengestellt. Anschließend wurden im Rahmen von Workshops mit den Unternehmenspartnern der Soll-Zustand genauer detailliert und Pilotprojekte als Annäherung und erste Umsetzung ausgewählt. Je Unternehmenspartner wurde dann das Vorgehen für ein Pilotprojekt unter Berücksichtigung der Gestaltungsempfehlungen geplant. Für die Planung der Technologieeinführungen wurden zudem ergänzend Erfolgsfaktoren aus der Implementierungsforschung berücksichtigt (für Details s. Zorn et al. 2020).

Eine erste Annäherung an den Soll-Zustand erfolgte dann in Pilotprojekten, für die begleitende Datenerhebungen konzipiert wurden. Auf Basis des erweiterten 3-SichtenModells sowie Erfolgsfaktoren für Implementierung wurde dazu ein Online-Fragebogen erstellt, der möglichst ökonomisch die wichtigsten Themen erfassen sollte. Diese umfassten Aufgabeninterdependenz, Teamstimmung sowie wahrgenommene Leistung (vgl. Abschn. 8.3.). Um erwartete Veränderungen in der Kommunikation zu erheben, wurden Kommunikationsanlässe und Abteilungszugehörigkeit der Kommunikationspartner in den Fragebogen aufgenommen.

Eines der Pilotprojekte konnte bereits umgesetzt werden, sodass die begleitende Datenerhebung für dieses Projekt abgeschlossen werden konnte. Befragt wurden dabei 5 Mitarbeitende aus dem Bereich Konstruktion und Entwicklung mittleren Alters mit mehrjähriger Betriebszugehörigkeit.

\subsection{Fallbeispiele}

Die Ergebnisse werden in diesem Abschnitt in Form von Fallbeispielbeschreibungen vorgestellt. Ebenen des 3-Sichten-Modells sowie daraus entnommene Kernbegriffe sind kursiv gesetzt, um den Lesenden die Zuordnung zu erleichtern. 


\subsubsection{Virtuelle Inbetriebnahme}

Das erste Fallbeispiel befasst sich mit der Einführung der virtuellen Inbetriebnahme in Unternehmen A. Üblicherweise entfällt der größte Anteil der Qualitätssicherung für Maschinen oder Anlagen auf die konventionelle Inbetriebnahme am Ende des Produktentwicklungsprozesses (Zäh et al. 2006). Der Begriff „Inbetriebnahme“ bezeichnet die vollständige Prüfung einer Maschine oder Anlage hinsichtlich Funktionalität und fehlerfreier Abläufe. Dabei werden alle Abläufe und Funktionen geprüft, die für den späteren Betrieb der Anlage notwendig sind. Die konventionelle Inbetriebnahme ist in Unternehmen A einerseits durch eine starke Kapazitätslimitation gekennzeichnet, andererseits aber von großer Bedeutung für die Qualitätssicherung. Während eine konventionelle Inbetriebnahme erst am Ende des Produktentstehungsprozesses nach abgeschlossener Entwicklung der Teilsysteme möglich ist und Fehler daher erst spät festgestellt werden können, erlaubt die virtuelle Inbetriebnahme bereits eine Prüfung von Anlagenteilen während der Entwicklung. Dies ermöglicht es, Fehler früh zu identifizieren und direkt zu beheben, bevor das erste Teil der Maschine physisch gefertigt wurde.

Der aktuelle Status quo ist auf Prozessebene ein sequenzieller Entwicklungsprozess. Ein Auftrag (Entwicklungsaufgabe)wird nacheinander von verschiedenen Entwicklungsdisziplinen(z. B. Mechanik, Hydraulik, Steuerungstechnik) bearbeitet. Bis auf kleinere Anpassungen werden in der Regel fertige Zwischenprodukte an die nächste Abteilung weitergegeben. Die einzelnen Teilsysteme werden erst gegen Ende des Produktentwicklungsprozesses zusammengefügt. Innerhalb des Entwicklungsprozesses haben die Mitarbeitenden klare Rollen und in sich abgeschlossene Aufgaben, d. h. innerhalb eines Projektes liegen klare Zuständigkeiten vor. Der Informationsfluss ist zu bestimmten Phasen (z. B. Projektauftakt) prozessbedingt strukturiert, während der Projektbearbeitung eher bedarfsorientiert (z. B. kurzfristige Abstimmungen zwischen Abteilungen). Aufgrund der klaren Rollenzuteilung funktioniert auch spontane Informationsweitergabe in der Regel gut. Auf Methodenebene stehen den Mitarbeitenden für Entwicklungsaktivitäten verschiedene Methoden, Kommunikationsmedien und Technologien zur Verfügung, die überwiegend nach situativer Passung ausgewählt und angewandt werden. Die Nutzung weist dabei einen engen Zusammenhang zu den Kompetenzen der Mitarbeitenden auf. Als Kommunikationsmittel werden persönlichere Kommunikationswege (z. B. Abteilungsbesprechungen, Anrufe) bevorzugt, was durch das Arbeiten an einem zentralen Standort ermöglicht wird. Virtuelle Kommunikationsmittel (z. B. Videokonferenzen, Chats) sind einerseits durch die räumliche Nähe unüblich und andererseits entscheiden sich die Mitarbeitenden auch eher aktiv für andere, als persönlicher wahrgenommene Kommunikationswege. Ein Mitarbeiter aus dem Unternehmen beschrieb dies im Interview so: „wir sitzen alle (...) auf einer Ebene, räumlich sehr nah beieinander, $d$. h. man spricht sich einfach im persönlichen Gespräch $a b$ “. Zu dieser allgemeinen, unternehmensweiten Präferenz von persönlichen Kommunikationswegen bevorzugen zudem Mitarbeitende je nach Gegenüber und Anlass unterschiedliche 
Kommunikationsmittel. Diese Tendenzen könnten ohne die Betrachtung des Entwicklungsteams, insbesondere der Teamkognitionen, nicht im 3-Sichten-Modell verortet werden. Die Wahl des Kommunikationsmittels ist in diesen Fällen dadurch motiviert, wie die Mitarbeitenden mit einer anderen Person effektiv kommunizieren können, bspw. ob eine Informationsweitergabe per E-Mail oder eine Abstimmung per Anruf sinnvoller ist. Dies entspricht teambezogenen geteilten mentalen Modellen (vgl. Cannon-Bowers et al. 1993). Auf Kompetenzebene sind bei den Mitarbeitenden insgesamt die notwendigen fachlichen Kenntnisse vorhanden, um ihre Aufgaben auszuführen. Unterschiede gibt es hauptsächlich hinsichtlich methodischer und überfachlicher Kompetenzen. Dies zeigt sich in erster Linie im unterschiedlichen Einsatz von Technologien, Methoden und Hilfsmitteln, d. h. auf Methodenebene. Weitere Unterschiede sind im Verhalten der Mitarbeitenden in der Zusammenarbeit sichtbar, also zum Teil auf Prozessebene z. B. inwiefern Mitarbeitende proaktiv Abstimmungen mit anderen Abteilungen anstoßen (Informationsfluss). Ein Teil der überfachlichen Kompetenzen zielt allerdings eher auf die funktionale Interaktion im Team ab (z. B. Konflikte sachlich zu lösen). Ihre Auswirkungen werden erst durch die Inklusion des Entwicklungsteams im Modell sichtbar.

Der Soll-Zustand sieht die Einbindung der virtuellen Inbetriebnahme vor und führt deswegen zu verschiedenen Änderungen. Um die Vorteile der virtuellen Inbetriebnahme voll auszuschöpfen, muss auf Prozessebene eine Veränderung von sequenziellen hin zu parallelen Prozessen vorgenommen werden. Nur so ist die erforderliche Integration für die virtuelle Inbetriebnahme möglich, da alle Teilsysteme für die virtuelle Inbetriebnahme eine gewisse, abgestimmte Entwicklungsreife aufweisen müssen. Dies geht allerdings auf Prozessebene mit einer Steigerung der Komplexität einher, zwischen einzelnen Entwicklungsaktivitäten entstehen mehr Wechselwirkungen. Werden in einer Abteilung Änderungen am ursprünglichen Plan vorgenommen, müssen diese Änderungen schnellstmöglich an die anderen Abteilungen weitergegeben werden, damit diese direkt berücksichtigt werden können und nicht erst bei der virtuellen Inbetriebnahme auffallen. Auf Methodenebene wird zur Umsetzung der virtuellen Inbetriebnahme einerseits ein digitales Hilfsmittel, in diesem Fall eine Software, benötigt, die eine virtuelle Überprüfung von Funktionalitäten ermöglicht. Andererseits muss die genutzte Software mit den bereits vorhandenen Programmen kompatibel sein und über passende Schnittstellen verfügen, da aufwendige Dateikonvertierungen Zeitverzögerungen verursachen würden. Des Weiteren werden Methoden und weitere Hilfsmittel benötigt, die bei der Bewältigung der neuen Komplexität unterstützen, z. B. Methoden zur strukturierten Problemlösung oder Hilfsmittel wie Prozessvisualisierungen, Checklisten oder Übersichten $\mathrm{zu}$ Verantwortlichkeiten. Auf Kompetenzebene schlagen sich komplexere Entwicklungsprozesse und neue Methoden und Technologien in neuen Anforderungen für die Mitarbeitenden nieder. Die Mitarbeitenden müssen die methodischen Kompetenzen zur Bedienung und Anwendung der neuen (digitalen) Hilfsmittel und Methoden entwickeln, die sie zur Umsetzung der virtuellen Inbetriebnahme benötigen (z. B. Simulationssoftware). Mitarbeitende brauchen darüber hinaus auch ein Verständnis davon, wie Änderungen, die sie vornehmen, andere Teilsysteme beeinflussen 
und welche Informationen sie daher an wen und bis wann weiterleiten müssen. Nur so können sie dann die richtigen Kollegen und Kolleginnen frühzeitig informieren oder aktiv die Abstimmung mit ihnen suchen. Dazu benötigen die Mitarbeitenden wiederum entsprechende soziale Kompetenzen, um die notwendigen Abstimmungen erfolgreich durchzuführen (z. B. Kommunikationskompetenz). In einem Interview beschrieb eine Führungskraft es so, dass erfolgreiche Mitarbeitende ein Problem aus verschiedenen fachlichen Perspektiven betrachtet und dann gut „auf den Punkt gebracht“ haben. Auch hier lässt sich die Auswirkung der Kompetenzen zur erfolgreichen Kommunikation nur durch die Erweiterung um das Entwicklungsteam im 3-Sichten-Modell einordnen. Geteilte mentale Modelle zur Aufgabe, zur Ausrüstung, zur Teaminteraktion und zu den Teammitgliedern (vgl. Cannon-Bowers et al. 1993) könnten hier zur effizienten, erfolgreichen Abstimmung beitragen. Ein fiktives Beispiel zur Veranschaulichung: Mitarbeiter X muss eine Änderung an der Form der Maschine vornehmen, die auch den Innenraum der Maschine betreffen. Er weiß, dass diese Änderungen Auswirkungen auf die pneumatische Konstruktion und die geplanten elektrischen Bauteile (z. B. Kabellängen, Sensorplatzierungen) haben könnte. Deswegen gibt er diese Information direkt telefonisch seinen beiden Kollegen Y (Pneumatik) und Z (Elektrik) weiter, weil er weiß, dass sie dann sofort ihre eigenen Bauteile überprüfen und anpassen können. Im Anschluss schreibt X noch eine E-Mail an seine Kollegen und legt diese auch zusammen mit den übrigen Produktdaten ab, damit die Änderungen später nachvollziehbar sind.

Als realistischen Umfang für ein zeitlich begrenztes Pilotprojekt wurde die virtuelle Inbetriebnahme eines Bauteils als Entwicklungsaufgabe festgelegt. Das ausgewählte Bauteil war Teil eines Weiterentwicklungsprojektes, um die Effizienz und Funktionalität der Maschine zu optimieren. Diese Entwicklungsaufgabe war daher nicht mit einem konkreten Kundenauftrag oder einer Deadline verbunden. Es wurden wie im Unternehmen üblich klare Rollen und Zuständigkeiten für das Pilotprojekt definiert, sodass die Mitarbeitenden möglichst optimale Voraussetzungen hatten, um mit dem neuen Prozess und den neuen Methoden und Hilfsmittel zurechtzukommen (s. auch Ruel et al. 2007). Des Weiteren wurde entschieden, die beteiligten Mitarbeitenden von anderen Aufgaben so weit zu entlasten, dass sie ausreichend Zeit hatten, das Pilotprojekt zu bearbeiten. Zudem wurden die Mitarbeitenden ermutigt, die Aufgabe zusammen zu bearbeiten, um so die Abstimmung zu vereinfachen und gegenseitige Unterstützung der Teammitglieder zu ermöglichen. Durch die gemeinsame Bearbeitung war es möglich, neue Aufgabeninterdependenzen direkt zu erkennen und so potenziell auch ein gemeinsames mentales Modell der virtuellen Inbetriebnahme zu entwickeln (s. Mathieu et al. 2000).

Während der Vorbereitung des Pilotprojektes ergaben sich zwei besondere Herausforderungen in Bezug auf die Software für die virtuelle Inbetriebnahme: Einerseits konnte aufgrund einer fehlenden Entwicklungslizenz keine Schnittstelle zwischen der Simulationssoftware und der in Unternehmen A verwendeten CAD-Software aufgebaut werden (Methodenebene). Deswegen war eine Behelfslösung nötig, die für die beteiligten Mitarbeitenden mit erheblich höherem Aufwand verbunden war. Andererseits war für die Bedienung der Simulationssoftware Programmieren notwendig, während die 
meisten Mitarbeitenden eher die Bedienung über Buttons gewöhnt waren (Kompetenzebene). Auch dies war wieder mit höherem Aufwand für die beteiligten Mitarbeitenden verbunden (ausführlicher dazu Paulsen et al. 2020).

Zur Umsetzung des Pilotprojekts arbeiteten die Mitarbeitenden kontinuierlich an der virtuellen Inbetriebnahme des Bauteils, häufig auch als Entwicklungsteam gemeinsam in einem Raum. In der Begleitbefragung zeigte sich, dass der negative Affekt über die gesamte Zeit des Pilotprojektes eher konstant niedrig ausgeprägt blieb, während der positive Affekt über die Zeit stärkere Variationen zeigte, aber insgesamt auf mittlerem bis niedrigem Niveau lag. Die Variationen zeigten keinen systematischen Zusammenhang in der virtuellen Inbetriebnahme (z. B. deren Start oder zwischen Wochen der Bearbeitung und Nicht-Bearbeitung). Dies passt zu Forschungsbefunden, dass positive und negative Teamstimmung unterschiedliche Merkmale sind (z. B. Paulsen et al. 2016). Der niedrige negative Affekt lässt vermuten, dass die Mitarbeitenden trotz des höheren Aufwandes keine ablehnende Haltung gegenüber der virtuellen Inbetriebnahme entwickelt haben. Ein Scheitern der virtuellen Inbetriebnahme aufgrund Ablehnung der Mitarbeitenden erscheint nach diesem Stand unwahrscheinlich (s. Bondarouk und Sikkel 2007). Aufgabeninterdependenz zeigte einen annähernd spiegelbildlichen Verlauf zu positivem Affekt, d. h. sinkender positiver Affekt trat zeitgleich mit steigender Aufgabeninterdependenz auf und umgekehrt. Wahrgenommene Leistung wies einen ähnlichen Verlauf wie positiver Affekt auf, lag allerdings eher auf hohem Niveau. Diese Ähnlichkeiten in den Verlaufsmustern zeigen einen möglichen Zusammenhang zwischen positivem Affekt, Aufgabeninterdependenz und Teamleistung auf, wie er in der Forschung schon für Affekt und Interdependenz (Bartel und Saavedra 2000) bzw. Affekt und Leistung (z. B. Knight und Eisenkraft 2015) gefunden wurde. Insgesamt konnte auf Basis der Stimmungs- und Leistungsbewertungen kein Interventionsbedarf festgestellt werden. Bezüglich der Kommunikation machten Informationsaustausch und Koordination zwar vergleichsweise hohe Anteile der Kommunikationsanlässe aus, zeigten aber keine Verbindung mit Reduzierungen im positiven Affekt oder der wahrgenommenen Leistung. Rückfragen und Konfliktmanagement bleiben konstant auf einem niedrigen Niveau (weniger als $10 \%$ der Kommunikationsanlässe in einer Woche). Die abteilungsübergreifende Kommunikation war zwar intensiver als die abteilungsinterne Kommunikation, trat aber insgesamt seltener auf als die abteilungsinterne Kommunikation. Das Team konnte die virtuelle Inbetriebnahme des Bauteils trotz des höheren Aufwandes erfolgreich abschließen und bewertete das Konzept der virtuellen Inbetriebnahme insgesamt positiv.

Insgesamt zeigt sich, dass umfangreiche Änderungen auf Prozess-, Methoden- und Kompetenzebene notwendig sind, um das Digitalisierungsvorhaben der virtuellen Inbetriebnahme umzusetzen. Allerdings sind die notwendigen Änderungen, wenn sie umfassend und einschließlich der Konsequenzen und Auswirkungen auf andere Bereiche analysiert werden, für Unternehmen A umsetzbar. Mithilfe des 3-Sichten-Modells konnten Erfolgsfaktoren wie die sehr gute Rollenklärung sichtbar gemacht werden, die dann explizit für die Planung der Pilotprojekte berücksichtigt werden konnten. Durch 
die Erweiterung um die Teamperspektive konnten einerseits die Interaktion der Mitarbeitenden abgebildet werden, z. B. indem geteilte mentale Modell zur Erklärung der situationsspezifischen Auswahl von Kommunikationsmitteln je nach Gesprächsbeteiligten und Ziel herangezogen werden können. Da diese Merkmale nun im Modell sichtbar gemacht werden, können sie auch in der Gestaltung adressiert werden, bspw. mit speziell gestalteten Interventionen (s. Ellwart et al. 2015). Teamemotion kann aufgrund der Verbindung mit Aufgabeninterdependenz und Leistung einerseits und der guten Erfassbarkeit andererseits als frühzeitiger Indikator für Interventionsbedarf genutzt werden, bspw. über ein sog. Stimmungsbarometer (s. auch Kauffeld und Güntner 2018). Durch den Zusammenhang von Teamstimmung bzgl. neuer Technologien und deren erfolgreicher Implementierung (s. Bondarouk und Sikkel 2007) kann die Beobachtung der Stimmung bei Digitalisierungsvorhaben besonders bedeutsam sein.

\subsubsection{Standortübergreifende Produktentwicklung}

Das zweite Fallbeispiel betrifft die standortübergreifende Produktentwicklung in Unternehmen B, zwischen dem Hauptstandort des Unternehmens in Deutschland und dem zu diesem Zeitpunkt im Aufbau befindlichen Standort in Indien. Während der indische Standort aufgebaut wurde, übernahmen die Mitarbeitenden in Deutschland die Bearbeitung von Kundenaufträgen für den indischen Markt. Dies führte zu einer höheren Auslastung der Mitarbeitenden am deutschen Standort, die die Kapazitäten für Neuentwicklungen stark reduzierte. Mit der Etablierung einer eigenen Entwicklungsabteilung am indischen Standort sollten indische Mitarbeitende durch die standortübergreifender Zusammenarbeit zur Bearbeitung der Aufträge von lokalen Kunden befähigt und gleichzeitig Mitarbeitende am deutschen Standort entlastet werden. Durch die gleichmäßigere Auslastung der Konstruktionsabteilungen wurde zudem eine schnellere Bearbeitung von Aufträgen erwartet.

Die Ausgangssituation in Unternehmen B ist gekennzeichnet durch geringe Zusammenarbeit zwischen den beiden Standorten. Frühere Versuche, in der Produktentwicklung standortübergreifend zusammenzuarbeiten, wurden dabei meist nach kurzer Zeit eingestellt, da sie insgesamt zu einer Verlängerung der Auftragsbearbeitung geführt haben. Bei der Betrachtung der Prozessebene wurde festgestellt, dass diese Verzögerungen systematisch in der Phase der Konstruktion auftraten. Im Gegensatz zur lokalen Produktentwicklung kamen bei der standortübergreifenden Zusammenarbeit vermehrte Bearbeitungsschleifen bei der Anfertigung der digitalen3-D-Konstruktionsmodelle vor. Als Resultat erstellten die Mitarbeitenden am Hauptstandort meist die Konstruktion selbst, sodass die erhoffte Arbeitsentlastung häufig nicht gegeben war. Eine Ursache für die Verzögerungen in der Auftragsbearbeitung konnten auf Methodenebene aufgedeckt werden. Zum einen verfügten beide Standorte über unterschiedliche Versionen der Konstruktionssoftware, sodass Datenaustausch grundsätzlich nur eingeschränkt möglich war. Zum anderen standen E-Mails als einzige Möglichkeit zum 
sicheren Datenaustausch zur Verfügung, wobei aber aufgrund der Dateigrößen keine Konstruktionsdateien ausgetauscht werden konnten. Stattdessen wurden PDF-Dateien der Konstruktionen per Mail ausgetauscht, Änderungsbedarf konnten nur per Hand und als Kommentar auf ausgedruckten Dokumenten vermerkt werden. Nach Abschluss der Konstruktion mussten zudem die Parameter des digitalen Modells in die Konstruktionssoftware eingepflegt werden, wodurch weiterer Zusatzaufwand für die Mitarbeitenden entstand. Eine Person fasste die Situation so zusammen: „Das geht dann so weit, dass (...) wenn die das machen und ich muss das alles nachplegen, dann kann ich es auch gleich alleine machen“. Ein weiterer Problembereich konnte auf Kompetenzebene identifiziert werden. Die Mitarbeitenden an beiden Standorten verfügten zwar über die notwendigen fachlichen und methodischen Kompetenzen für die Konstruktion von Produkten mittels CAD-Software, allerdings fehlte den Mitarbeitenden in Indien unternehmensspezifische Expertise zu Produkten und Prozessen. Am Hauptstandort haben sich Konventionen und Standards in Bezug auf bestimmte Produkte (z. B. zu bevorzugtem Material oder Abmessungsverhältnissen) etabliert, die neuen Mitarbeitenden im Zuge der Einarbeitung vermittelt wurden. Der neu etablierten Konstruktionsabteilung in Indien stand dieses Wissen nicht zur Verfügung, da die Mitarbeitenden in Indien nicht die gleiche Einarbeitung erhielten und vorhandene Hilfsmittel (wie z. B. Checklisten oder Leitfäden) nur in deutscher Sprache verfasst waren. Die Mitarbeitenden an den beiden Standorten verfügten also über unterschiedliche mentale Modelle, wie eine Entwicklungsaufgabe zu bearbeiten war und welche Informationen an den anderen Standort weitergegeben werden mussten. $\mathrm{Zu}$ dieser Differenz im Wissensstand kamen Herausforderungen in der Kommunikation, die sich negativ auf den Wissensaustausch auswirkten. Dabei verunsicherten die Kommunikation in einer Fremdsprache sowie die wahrgenommenen kulturellen Unterschiede die Mitarbeitenden, sodass Informationsaustausch in erster Linie nur zu Weitergabe von Aufgaben und Änderungsbedarf stattfand. Die Projektteams waren dabei mit den typischen Herausforderungen interkultureller virtueller Teams konfrontiert (dazu ausführlicher Schulze und Krumm 2017): Durch den Zeitunterschied stand nur ein relativ kurzes Zeitfenster für direkte, synchrone Kommunikation über digitale Medien zur Verfügung, die anfällig für kulturelle und sprachliche Missverständnisse ist. In einem Interview beschrieb eine Person, dass es schwer zu erkennen wäre, ob die Kollegen am anderen Standort bei einer Besprechung wirklich keine Fragen mehr hätten oder diese aus Höflichkeitsgründen nur nicht äußern würden. Wenn es dann anschließend zu Abweichungen käme, würde man dann aus Unsicherheit dazu übergehen, in der folgenden Zusammenarbeit grundsätzlich alles ganz genau zu kontrollieren. Durch solche Erlebnisse entwickelte sich das notwendige Vertrauen zwischen den Teammitgliedern noch langsamer, als dies ohnehin schon bei fehlenden Möglichkeiten zur face-to-face-Interaktion der Fall ist (s. Handke und Kauffeld 2019). Insgesamt stellt die geplante Zusammenarbeit aufgrund der hohen Komplexität, der Einschränkung durch virtuelle Kommunikationsmittel und durch Neuartigkeit des Vorhabens noch fehlende Ressourcen besondere Anforderungen an Arbeitsgestaltung 
(s. Handke et al. 2020). Für die Erreichung des Soll-Zustands und die Umsetzung des Pilotprojekts wurde daher umfangreicher Vorbereitungsbedarf erwartet.

Der angestrebte Soll-Zustand sieht vor, dass Gesamtprodukte standortübergreifend entwickelt werden können. Über die Entwicklungsaufgabe werden genaue Rahmenbedingungen wie Deadlines und Spezifikationen des Ergebnisses (Produkt, Abgabeformat, Zusatzdateien usw.) vorgegeben. Die Zusammenarbeit soll nach einem einheitlichen Prozess erfolgen, der auch Rollen und Informationsfluss regelt. Mittels stabiler, sicherer Datenverbindung sowie kompatibler Technologie sollen Konstruktionsdateien direkt ausgetauscht und bearbeitet werden. Des Weiteren sollen allen Mitarbeitenden deutsch- und englischsprachige Hilfsmittel wie Leitfäden, Konstruktionskataloge oder Checklisten zur Verfügung stehen. Zudem soll es immer wieder Möglichkeiten für Mitarbeitende aus Indien geben, von deutschen Mitarbeitenden eingearbeitet $\mathrm{zu}$ werden und damit alle nötigen Kompetenzen $\mathrm{zu}$ entwickeln. Dabei soll auch der informelle Austausch zwischen den Mitarbeitenden gefördert werden, um so Vertrauen auf- und Hemmungen abzubauen.

Zur Erreichung dieses Soll-Zustands ist eine schrittweise Ausweitung vorgesehen. $\mathrm{Zu}$ Beginn werden kleinere Bauteile standortverteilt konstruiert. Der Umfang wird schrittweise hochskaliert, sobald eine festgelegte Anzahl an standortübergreifender Entwicklungssaufgaben erfolgreich bearbeitet wurde. Dazu wurden bisher die notwendigen technischen Voraussetzungen geschaffen (u. a. Datenverbindung, kompatible Software). Zusätzlich ist die Nutzung eines digitalen Hilfsmittels (Powl-Tool) zur Prozessvisualisierung (für Details s. Baschin et al. 2019) vorgesehen. Ein Mitarbeiter aus Indien war zudem bereits am Hauptstandort und bearbeitete dort zusammen mit deutschen Kollegen und Kolleginnen typische Aufgaben. Einerseits führte dies schnell zum erwünschten Kompetenzerwerb bzgl. unternehmensspezifischer Besonderheiten, andererseits entwickelte sich auch der erhoffte informelle Austausch. Die Mitarbeitenden kommunizierten bereitwilliger und informeller auf Englisch. Über den Verlauf der Zusammenarbeit wird für die beteiligten Mitarbeitenden ein weiterer Kompetenzzuwachs in Bereichen wie interkulturelle Zusammenarbeit, Vertrauensaufbau in virtuellen Teams und Selbstmanagement (z. B. strukturiertes Arbeiten und Dokumentieren) erwartet.

Zusammenfassend lässt sich für dieses Fallbeispiel festhalten, dass ganzheitlichere Ansätze zur Problemanalyse eine genauere Auflösung von komplexen Systemen bieten können. Es zeigt sich, dass das 3-Sichten-Modell nicht nur zur Analyse von Ist- und Soll-Zustand geeignet ist, sondern ebenfalls zur Analyse vergangener (erfolgreicher oder gescheiterter) Veränderungen genutzt werden kann. Kann auf solche Informationen zurückgegriffen werden, können diese verwendet werden, um relevante Barrieren für das geplante Vorhaben zu identifizieren. Eine genauere Aufstellung von Hindernissen und Herausforderungen erlaubt wiederum eine spezifischere Planung, wie diese erfolgreich bewältigt werden könnten. So konnte das Problem der unterschiedlichen mentalen Modelle bezüglich der Bearbeitung von Entwicklungsaufgaben durch die gemeinsame Einarbeitung gelöst werden (s. auch Ellwart et al. 2015; Mathieu et al. 2000). Zudem 
förderte die face-to-face-Zusammenarbeit zu Beginn den Beziehungs- und Vertrauensaufbau, der die weitere, dann virtuelle Zusammenarbeit erleichtern kann (Kauffeld et al. 2016).

\subsection{Empfehlungen für die Gestaltung der Digitalisierung in der Produktentwicklung}

Aus den beiden Fallbeispiele können zwei zentrale Erkenntnisse gezogen werden, wie Digitalisierungsvorhaben für Produktentwicklungsprojekte gestaltet werden können.

Die erste Erkenntnis betrifft vor allem die Planung von Digitalisierungsvorhaben bzw. die dazu nötigen Vorbereitungen. Zur erfolgreichen Gestaltung ist eine sorgfältige Analyse als erster Schritt sinnvoll. Eine sorgfältige Analyse umfasst Ist- und Soll-Zustand auf verschiedenen Ebenen, um Wechselwirkungen berücksichtigen zu können (s. auch Davis et al. 2014; Negele et al. 1997). Dies wird bspw. im Fall von Unternehmen B deutlich: Der Prozess für die standortverteilte Zusammenarbeit wurde geplant, da fachliche Kompetenzen zur Bearbeitung der Entwicklungsaufgabe und Kommunikationsmittel für den Austausch vorhanden waren. Trotzdem verliefen die ersten Versuche nicht erfolgreich, die beteiligten Mitarbeitenden stießen immer wieder auf Probleme, sodass die Zusammenarbeit schließlich jedes Mal wieder eingestellt wurde. Die Anwendung des 3-Sichten-Modells führte hier dazu, dass mehr potenzielle Hindernisse identifiziert und entsprechende Gegenmaßnahmen geplant werden konnten. Insbesondere aus den früheren Versuchen konnten dabei viele Erkenntnisse zu Hindernissen gezogen werden. Die Vorbereitung des Pilotprojektes verlief so bereits vielversprechender als bei vorherigen Versuchen. Dagegen konnten für Unternehmen A mittels des 3-SichtenModells eher Erfolgsfaktoren aus dem üblichen Vorgehen der Mitarbeitenden abgeleitet werden, die für die Planung des Pilotprojektes berücksichtigt wurden und während der Durchführung zur erfolgreichen Bearbeitung beigetragen haben. Informationen zu vergangenen Versuchen oder ähnlichen, erfolgreich verlaufenen Veränderungen erleichtern es demnach, neben möglichen Herausforderungen auch die praktisch vorkommenden Hindernisse und Probleme zu adressieren und so Digitalisierungsvorhaben erfolgreich umzusetzen.

Umfassende Betrachtungen erhöhen allerdings den Grad an Komplexität erheblich, was unter anderem die Verarbeitung einer großen Menge an Informationen mit sich bringt. Strukturierung ist eine Möglichkeit, damit umzugehen (s. zusammenfassend Antoni und Ellwart 2017), wobei Modelle eine Möglichkeit zur Strukturierung von Informationen darstellen. Unternehmen B verfügte bspw. über Informationen darüber, wo genau in der standortübergreifenden Zusammenarbeit Probleme auftraten und welche Ursachen diese hatten. Diese Informationen wurden aber nicht systematisch miteinander verbunden, sodass kaum Erkenntnisse daraus gezogen werden konnten. Durch die systematische Strukturierung anhand des 3-Sichten-Modells wurden Zusammenhänge 
deutlicher, entsprechend konnten konkretere Maßnahmen geplant werden. Hierbei ist wichtig, dass bei Nutzung des Modells eine konkrete Zuordnung von Informationen o. ä. möglich ist (s. Ehrlenspiel und Meerkamm 2013). Beispielsweise wäre das MTO-Modell (n. Ulich 2013) für organisationsweite Änderungen geeignet, aber für den konkreten Fall eines Produktentwicklungsprojektes zu umfangreich und ggf. auch zu abstrakt. Das ZOPH-Modell (n. Negele et al. 1997) ist zwar für die Produktentwicklung gedacht, fasst aber Mitarbeitende, organisationale und Kontextfaktoren zusammen. Eine Berücksichtigung der Mitarbeitenden - oder des Teams - ist damit in der Praxis schwierig. Für das 3-Sichten-Modell konnten die zugrunde liegenden konzeptionellen Überlegungen (z. B. Bavendiek et al. 2018) sowie die praktische Anwendbarkeit für die Fallbeispiele bestätigt werden.

Die Anwendung von Modellen kann dabei auch durch digitale Hilfsmittel unterstützt werden (z. B. Negele et al. 1997). Für das 3-Sichten-Modell ist im Rahmen des Verbundprojektes ein digitales Hilfsmittel, das Powl-Tool, entwickelt worden, das neben der Prozessmodellierung direkt die Integration von Methoden, Hilfsmitteln und Kompetenzen in die jeweiligen Prozessschritte ermöglicht (Details s. Baschin et al. 2019; Zorn et al. 2020). Dies ist sowohl für bestehende als auch geplante Prozesse nutzbar. Zum Zeitpunkt der Verfassung dieses Beitrags ist eine eigenständige Testung des Powl-Tools noch in Vorbereitung. Erste Erfahrungen aus Workshops zur Vorstellung des Powl-Tools zeigten aber, dass eine Abbildung der Prozesse, Methoden und Kompetenzen in geeigneter Detaillierung möglich ist. Verschiedene Prozesse konnten in den Workshops zur Zufriedenheit beider Unternehmenspartner im Powl-Tool vollständig und genau abgebildet und mit Methoden und Kompetenzen verknüpft werden. Das Risiko einer zu allgemeinen Darstellung und damit eine mangelnde praktische Eignung (s. Ehrlenspiel und Meerkamm 2013) erscheint daher gering.

Durch die Erweiterung um die Teamperspektive kann das 3-Sichten-Modell Produktentwicklungsprojekte noch besser abbilden. Einerseits können mittels Teamkognitionen und Teamemotionen wichtige zusätzliche Wechselwirkungen sichtbar gemacht werden, bspw. wie geteilte mentale Modelle die Wahl von Kommunikationsmitteln beschreiben können oder wie unterschiedliche mentale Modelle die Zusammenarbeit erschweren. Andererseits stellen beide Faktoren wichtige Rahmenbedingungen für erfolgreiche Teamarbeit dar (z. B. Ellwart et al. 2015; Knight 2015). Zudem kann Teamarbeit durch Förderung geteilter mentaler Modelle (s. Ellwart et al. 2015) sowie durch frühzeitige Interventionen bei der Erhebung von Teamstimmung (s. Kauffeld und Güntner 2018) aktiv gestaltet werden. Für die virtuelle Zusammenarbeit ist dies umso wichtiger (s. Kauffeld et al. 2016; Schulze und Krumm 2017). Organisationen müssen hier vor allem gute Rahmenbedingungen, angefangen bei der nötigen technischen Infrastruktur, schaffen, während Teams insbesondere einen Fokus auf Beziehungsaufbau legen sollten, um die Einschränkungen digitaler Kommunikationsmedien zu kompensieren, und Individuen müssen ihre eigenen Kompetenzen für die verteilte Zusammenarbeit weiterentwickeln (s. Kauffeld 2020). 
Die zweite Erkenntnis betrifft die Umsetzung von Digitalisierungsprojekten, speziell die dafür meist erforderlichen Einführungsprozesse für neue Technologien (z. B. Software). Trotz nicht optimaler Voraussetzungen für die virtuelle Inbetriebnahme war dies nur mit geringer Belastung für die Mitarbeitenden verbunden. Das Konzept der virtuellen Inbetriebnahme wurde auch insgesamt positiv aufgenommen. Dies lag einerseits daran, dass die Mitarbeitenden zur Bearbeitung des Pilotprojektes von anderen Aufgaben freigestellt wurden und das Bauteil, das virtuell in Betrieb genommen wurde, eine Neuentwicklung war und somit nicht als Teil eines Auftrags mit einer Deadline verbunden war. Dies ist wichtig, da Technologieimplementierungen ausreichend Zeit benötigen (Bondarouk und Sikkel 2003b). Andererseits wurden bei der Durchführung des Pilotprojektes systematisch Erfolgsfaktoren aus der Implementierungsforschung in Großunternehmen in einer sinnvollen Reihenfolge angewandt (ausführlich dazu Zorn et al. 2020). Über die Berücksichtigung von Prozessen, Methoden, Kompetenzen, Entwicklungsaufgabe und -team hinaus wurden die Mitarbeitenden bei der Implementierung systematisch einbezogen. Abb. 8.3 stellt diesen Prozess in verallgemeinerter Form dar. Dieses Modell von Technologieimplementierung ist dabei besonders geeignet für den Teamkontext, da Gruppenlernphänomene aktiv genutzt werden, die zum Erfolg einer Technologieeinführung beitragen können (Bondarouk und Sikkel 2003a; Ruel et al. 2007).

Dieser Modellprozess beruht auf der Systematisierung von empirisch identifizierten Erfolgsfaktoren für Technologieimplementierung anhand der Beobachtungen im Fallbeispiel. Eine systematische empirische Überprüfung des Gesamtmodells steht noch aus. Für Anwender aus der Praxis bietet es trotzdem bereits wissenschaftlich fundierte Ansatzpunkte, die auch von kleineren Unternehmen mit knappen Ressourcen umgesetzt werden können.

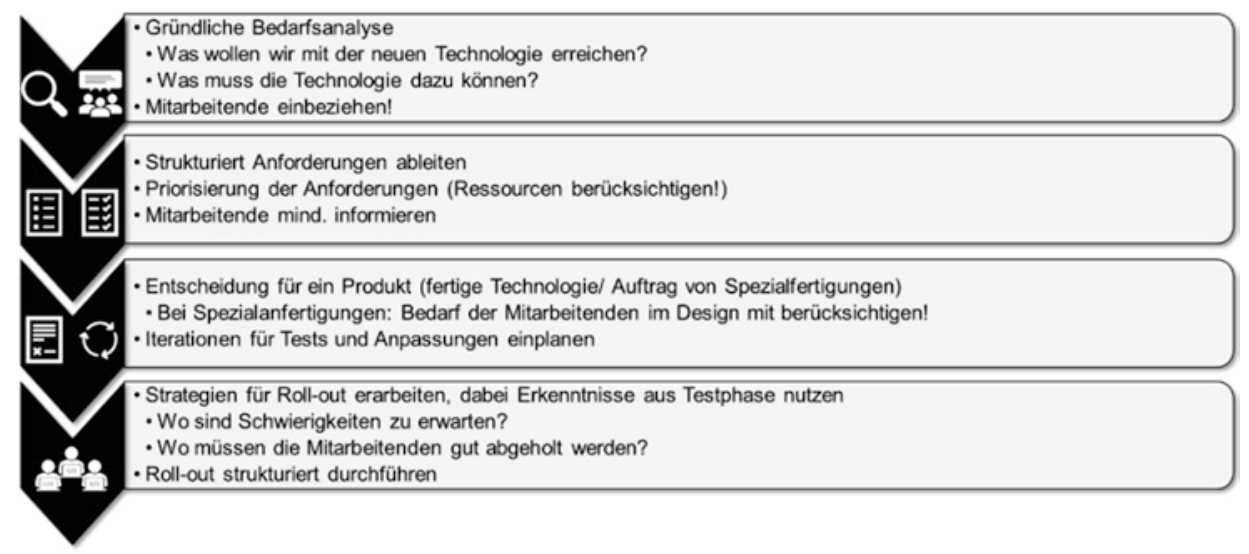

Abb. 8.3 Modellprozess für Technologieimplementierungen 


\section{Literatur}

Albers A, Burkardt N, Deigendesch T (2009) Vermittlung von Schlüsselqualifikationen am Beispiel des Karlsruher Lehrmodells für Produktentwicklung (KaLeP). In: Robertson-von Trotha CY (Hrsg) Schlüsselqualifikationen für Studium, Beruf und Gesellschaft. Technische Universitäten im Kontext der Kompetenzdiskussion Problemkreise der Angewandten Kulturwissenschaft. Universitätsverlag Karlsruhe, S 511-520

Antoni CH, Ellwart T (2017) Informationsüberlastung bei digitaler Zusammenarbeit - Ursachen, Folgen und Interventionsmöglichkeiten. GrInteraktOrg 48:305-315. https://dx.doi.org/10.1007/ s11612-017-0392-4

Badke-Schaub P, Frankenberger E (2004) Management Kritischer Situationen - Produktentwicklung erfolgreich gestalten. Springer, Berlin

Barsade SG (2002) The ripple effect: emotional contagion and its influence on group behavior. Adm Sci Q 47:644-675. https://dx.doi.org/10.2307/3094912

Bartel CA, Saavedra R (2000) The collective construction of workgroup moods. Admin-istrative Science Quarterly 45:197-231

Baschin J, Inkermann D, Vietor T (2019) Agile process engineering to support collaborative design. Procedia CIRP 84:1035-1040. https://dx.doi.org/10.1016/j.procir.2019.05.010

Bavendiek AK, Huth T, Inkermann D, Paulsen H, VietorT, Kauffeld S (2018) Collaborative design: linking methods, communication tools and competencies to processes. In: DS 92: Proceedings of the DESIGN 2018 15th International Design Conference, S 149-160

Bavendiek AK, Inkermann D, Vietor T (2017) Interrelations between processes, methods, and tools in collaborative design: A framework. In:Proceedings of the ICED'17/21st International Conference on Engineering Design, 8:349-358, The Design Society, Vancouver, Canada, August 21-25, 2017

Blumberg VSL, Kauffeld S (2020) Anwendungsszenarien und Technologiebewertung von digitalen Werkerassistenzsystemen in der Produktion. Gr Interakt Org 51:5-24

Bondarouk T, Sikkel N (2003a) Explaining groupware implementation through group learning. In: Kosrow-Pour M (Hrsg) Information Resource Management Association International Conference (IRMA'03). Idea Group Publishing, Hershey, PA, S 463-466

Bondarouk T, Sikkel K (2003b) Implementation of collaborative technologies as a learning process. In: Cano JJ (Hrsg) Critical reflections on information systems: a systemic approach. Idea Group Publishing, Hershey, PA, S 227-245

Bondarouk T, Sikkel K (2007) The relevance of learning processes for IT implementation. In: Khosrow-Pour M (Hrsg) Emerging information resources management and technologies. IGI Global, Hershey, PA, S 1-23

Boos M, Hardwig T, Riethmüller M (2016) Führung und Zusammenarbeit in verteilten Teams. Hogrefe, Göttingen

Born HJ (2018) Evolutionäre Geschäftsmodelle im Maschinenbau. In: Geschäftsmodell-Innovation im Zeitalter der vierten industriellen Revolution. Springer, Wiesbaden

Browning T, Fricke E, Negele H (2006) Key concepts in modeling product development processes. Syst Eng 9:104-128

Cannon-Bowers JA, Salas E, Converse SA (1993) Shared mental models in expert team decision making. In: Castellan NJ (Hrsg) Current issues in individual and group decision making. Erlbaum, Hillsdale, S 221-246

Cheshin A, Rafaeli A, Bos N (2011) Anger and happiness in virtual teams: emotional influences of text and behavior on others' affect in the absence of non-verbal cues. Organ Behav Hum Decis Process 116:2-16 
Crowder R, Bracewell R, Hughes G et al (2003) A future vision for the engineering design environment: a future sociontechnical scenario. In: International Conference on Engineering Design Iced 03 Stockholm. Stockholm

Cummings TG, Worley CG (2009) Organization development \& change. South-Western Cengage Learning, Mason

Davis MC, Challenger R, Jayewardene DNW, Clegg CW (2014) Advancing socio-technical systems thinking: a call for bravery. Appl Ergon 45:171-180. https://dx.doi.org/10.1016/j. apergo.2013.02.009

Eigner M, Roubanov D, Zafirov R (2014) Modellbasierte virtuelle Produktentwicklung. Springer, Berlin

Ellwart T, Happ C, Gurtner A, Rack O (2015) Managing information overload in virtual teams: effects of a structured online team adaptation on cognition and performance. Eur J Work Org Psychol 24:812-826

Ehrlenspiel K, Meerkamm H (Hrsg) (2013) Integrierte Produktentwicklung: Denkabläufe, Methodeneinsatz, Zusammenarbeit. Hanser, München

Franken S (2016) Arbeitswelt der Zukunft als Herausforderung für die Führung. In: Führen in der Arbeitswelt der Zukunft. Springer, Wiesbaden

Hagemann V, Kluge A, Ritzmann S (2011) High Responsibility Teams - Eine systematische Analyse von Teamarbeitskontexten für einen effektiven Kompetenzerwerb. Psychologie des Alltagshandelns 4:22-42

Handke L, Kauffeld S (2019) Alles eine Frage der Zeit? Herausforderungen virtueller Teams und deren Bewältigung am Beispiel der Softwareentwicklung. GrInterakt Org 50:33-41

Handke L, Klonek FE, Parker SK, Kauffeld S (2020) Interactive effects of team virtuality and work design on team functioning. Small Group Res 51:3-47. https://dx.doi. org/10.1177/1046496419863490

Hirsch-Kreinsen H, TenHompel M, Ittermann P, Dregger J, Niehaus J, Mättig B, Kirks T (2015) Digitalisierung von Industriearbeit: Forschungsstand und Entwicklungsperspektiven. Zwischenbericht des Projektes SoMaLi. Technische Universität Dortmund. https://www.wiwi.tudortmund.de/wiwi/de/forschung/gebiete/fp-hirschkreinsen/aktuelles/meldungsmedien/2015081 7Zwischenbericht-SoMaLI.pdf. Zugegriffen: 15. Juli 2019

Horváth I, Gerritsen, B, Rusák Z (2010) A new look at virtual engineering. In: Proceedings of Mechanical Engineering Conference, S 1-12

Huth T, Vietor T (2020) Systems Engineering in der Produktentwicklung: Verständnis, Theorie und Praxis aus ingenieurswissenschaftlicher Sicht. Gr Interakt Org 51:125-130. https://dx.doi. org/10.1007/s11612-020-00505-1

Inkermann D (2019) Towards Model-based Process Engineering. In: Proceedings of the 22nd International Conference on Engineering Design (ICED19), The Design Society, Delft, Netherlands, 5-8 August 2019

Kauffeld S (2007) Jammern oder Lösungsexploration - Eine sequenzanalytische Betrachtung des Interaktionsprozesses in betrieblichen Gruppen bei der Bewältigung von Optimierungsaufgaben. Z Arbeits- Organisationspsychol 51:55-67. https://doi.org/10.1026/0932-4089.51.2.55

Kauffeld S (2020) Räumlich und zeitlich verteilt mobil im Team arbeiten. In Kneips F, Pfaff H (Hrsg.) BKK Gesundheitsreport 2020 (in press)

Kauffeld S, Güntner AV (2018) Teamfeedback. In: Jöns I, Bungard W (Hrsg) Feedbackinstrumente im Unternehmen. Springer Gabler, Wiesbaden

Kauffeld S, Handke L, Straube J (2016) Verteilt und doch verbunden: Virtuelle Teamarbeit. GrInterakt Org 47:43-51

Kauffeld S, Paulsen H (2018) Kompetenzmanagement in Unternehmen. Kompetenzenbeschreiben, messen, entwickeln und nutzen. Kohlhammer, Stuttgart 
Knight AP (2015) Mood at the midpoint: affect and change in exploratory search over time in teams that face a deadline. Organ Sci 26:99-118

Knight AP, Eisenkraft N (2015) Positive is usually good, negative is not al-ways bad: the effects of group affect on social integration and task performance. J Appl Psychol 100:1214-1227

Lanting CJM, Lionetto A (2015) Smart systems and cyber physical systems paradigms in an IoT and Industrie/ie4.0 context. In: Paper presented at the 2nd International Electronic Conference on Sensors and Applications

Lipnack J, Stamps J (1998) Virtual teams. people working across boundaries with technology. Wiley, New York

Maguire M (2014) Socio-technical systems and interaction design - 21st century relevance. Appl Ergon 45:162-170

Mathieu JE, Heffner TS, Goodwin GF, Salas E, Cannon-Bowers JA (2000) The influence of shared mental models on team process and performance. J Appl Psychol 85:273-283

Negele H, Fricke E (1997) ZOPH - a systemic approach to the modeling of product development systems. INCOSE International Symposium 7:266-273

Pasmore W, Winby S, Mohrman SA, Vanasse R (2019) Reflections: sociotechnical systems design and organization change. J Change Manage 19:67-85

Paulsen HFK, Klonek FE, Schneider K, Kauffeld S (2016) Group affective tone and team performance: a week level study in project teams. Front Commun 1:7

Paulsen H, Zorn V, Inkermann D, Reining N, Baschin J, Vietor T, Kauffeld S (2020) Soziotechnische Analyse und Gestaltung von Virtualisierungsprozessen: Ein Fallbeispiel zur virtuellen Inbetriebnahme. Gr Interakt Org 51:81-93

Robin V, Rose B, Girard P (2007) Modelling collaborative knowledge to support engineering design project manager. Comput Ind 58:188-198. https://dx.doi.org/10.1016/j. compind.2006.09.006

Rouse WB, Morris NM (1986) On looking into the black box: prospects and limits in the search for mental models. Psychol Bull 100:349-363

Ruel HJ, Bondarouk TV, Van der Velde M (2007) The contribution of e-HRM to HRM effectiveness: results from a quantitative study in a Dutch Ministry. Empl Relat 29:280-291

Schleidt B, Eigner M (2010) Competency management approach for cross enterprise product design. In: Proceedings of the DESIGN 2010/11th International Design Conference. The Design Society, Glasgow, S 11183-1188. Dubrovnik, Croatia, 17-20. Mai 2010

Schulze J, Krumm S (2017) The "virtual team player": a review and initial model of knowledge, skills, abilities, and other characteristics for virtual collaboration. Org Psychol Rev 7:66-95

Sträter O, Bengler K (2019) Positionspapier Digitalisierung der Arbeitswelt. Z Arbeitswissenschaften 73:243-245

Talas Y, Gzara L, Le Dain M-A, Merminod V, Frank AG (2017) Which are the limitations of ICT tools for collaborative design with suppliers? In: Proceedings of the ICED'17/21st International Conference on Engineering Design, The Design Society 8:289-298, Vancouver, Canada, 21-25 August 2017

Törlind P, Larsson A (2002) Support for informal communication in distributed engineering design teams. In: Annals of 2002 International CIRP Design Seminar, Hong-Kong, 16-18 Mai 2002

Tuckman BW (1965) Developmental sequences in small groups. Psychol Bull 63:348-399

Ulich E (2013) Arbeitssysteme als soziotechnische Systeme: eine Erinnerung. J Psychol Alltagshandelns 6:4-12

Wallace K, Clegg C, Keane A (2001) Visions for engineering design: a multidisciplinary perspective. In: Proceedings of the ICED'01/13th International Conference on Engineering Design, Glasgow, UK, S 107-114, 21.-23. August 2001 
Zäh MF, Wünsch G, Hensel T, Lindworsky T (2006) Nutzen der virtuellen Inbetriebnahme: Ein Experiment. ZWF Z wirtschaftlichen Fabrikbetrieb 101:595-599

Zorn V, Baschin J, Berg A-K, Vietor T, Kauffeld S (2020) Digitale Hilfsmittel für digitale Arbeit? Ein praktischer Ansatz zur Etablierung eines digitalen Hilfsmittels für digitalisierungsbedingte Veränderungen. In: Gesellschaft für Arbeitswissenschaft e. V. Dortmund (Hrsg) Digitaler Wandel, digitale Arbeit, digitaler Mensch? (Beitrag A.9.7), GfA-Press, Dortmund

Open Access Dieses Kapitel wird unter der Creative Commons Namensnennung 4.0 International Lizenz (http://creativecommons.org/licenses/by/4.0/deed.de) veröffentlicht, welche die Nutzung, Vervielfältigung, Bearbeitung, Verbreitung und Wiedergabe in jeglichem Medium und Format erlaubt, sofern Sie den/die ursprünglichen Autor(en) und die Quelle ordnungsgemäß nennen, einen Link zur Creative Commons Lizenz beifügen und angeben, ob Änderungen vorgenommen wurden.

Die in diesem Kapitel enthaltenen Bilder und sonstiges Drittmaterial unterliegen ebenfalls der genannten Creative Commons Lizenz, sofern sich aus der Abbildungslegende nichts anderes ergibt. Sofern das betreffende Material nicht unter der genannten Creative Commons Lizenz steht und die betreffende Handlung nicht nach gesetzlichen Vorschriften erlaubt ist, ist für die oben aufgeführten Weiterverwendungen des Materials die Einwilligung des jeweiligen Rechteinhabers einzuholen.

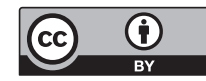

